\title{
FFT-ASSISTED ALGORITHMS FOR 3D LINE-CONTACT PROBLEMS
}

\author{
Sergiu Spinu ${ }^{1,2}$ \\ ${ }^{1}$ Department of Mechanics and Technologies, Stefan cel Mare University of Suceava, \\ $13^{\text {th }}$ University Street, 720229, Romania \\ 2 Integrated Center for Research, Development and Innovation in Advanced Materials, Nanotechnologies, and Distributed \\ Systems for Fabrication and Control (MANSiD), Stefan cel Mare University, Suceava, Romania \\ Corresponding author: Sergiu Spinu, sergiu.spinu@fim.usv.ro
}

\begin{abstract}
The line-contact is a particular type of contact with a contact length much greater than its width. Such contact scenarios can be treated in the frame of a twodimensional plane-strain problem if the contacting surfaces can be considered nominally smooth. However, surface irregularities inherent to any manufacturing technique lead to a discontinuous contact area that differs from the one derived on the basis of the smooth profile assumption. It is therefore tantalizing to pursue the solution of a line-contact problem using an intrinsically three-dimensional (3D) model, which can only be numerical due to lack of general analytical solutions in contact mechanics. Considering the geometry of the line-contact, a major challenge in its numerical modelling is that the expected contact area is orders of magnitude larger in one direction compared to the other. This may lead to an unreasonably large number of grids in the contact length direction, which translates to a prohibitive computational burden. An alternative approach, employed in this paper, is to treat the linecontact as non-periodic in the contact width direction, but periodic in the contact length direction, with a period equal to the window required to capture and replicate the surface specific texture. This periodicity encourages the contact problem solution by spectral methods based on the fast Fourier transform (FFT) algorithm. Based on this idea, two methods are derived in this paper from the existing Discrete Convolution Fast Fourier Transform (DCFFT) technique, which was previously developed for purely nonperiodic contact problems. A first algorithm variant employs a special padding technique for pressure, whereas a second one mimics the contribution of multiple pressure periods by summation of the influence coefficients over a domain a few times larger than the target domain. Both techniques are validated against the existing analytical Hertz solution for the line-contact and a good agreement is found. The advanced methods seem well adapted to the simulation of contact problems that can be approximated as periodic in one direction and non-periodic in the other.
\end{abstract}

Key words: elastic contact, fast Fourier transform, linecontact, convolution

\section{INTRODUCTION}

Line-contacts appear in engineering applications, such as cam-roller follower systems and spur gears, as well as in laboratory experimental rigs, in pairs like the cylinder-on-flat and the block-on-chain. For a convenient mathematical treatment, the line-contact problems are usually reduced to a plane-strain model and is solved by two-dimensional analytical or numerical endeavours [1-9]. As in contact mechanics the number of analytical solutions is reduced to a few, with the Hertz contact as a notable exception, numerical analysis of $3 \mathrm{D}$ contact problems is increasingly used to simulate the contact behaviour of engineering components and to promote the understanding of the contact processes.

Moreover, engineering surface may present an intentional treatment, such as texture or coating, together with undesirable topography perturbations such as roughness and superficial damages. In the numerical simulation, a specific minimum grid step may be required to capture the roughness essential features and the specifics of the textured surface. Whereas the finite element method works well with adaptive meshes, the modern semi-analytical methods that promise greater computational efficiency [10] require mesh to be uniform, although the step length can be different on any of the two tangential directions. Important computational time savings were achieved by employing spectral methods assisted by the fast Fourier transform (FFT) algorithm, which handles the calculation of the discrete Fourier transform and thus allows for efficient transfer to and from the space domain to the frequency domain.

The spectral analysis of contact processes pioneered with the work of Ju and Farris [5], who employed the continuous convolution theorem in the study of 2D contact problems. The consolidation of an efficient method for the study of non-periodic contact problems was achieved in $[11,12]$, i.e., the Discrete Convolution 
Fast Fourier Transform (DCFFT). Comprehensive study of the errors due to discretization in the space and in the frequency domain were reported in $[12,13]$. The DCFFT was later coupled [14] with the correlation theorem in the calculation of the elastic fields due to eigenstrains in a half-space, thus enabling a numerical solution to the elastic-plastic contact problem [15]. The elastoplastic contact of nominally flat surfaces was also studied [16] by FFT-based numerical methods. More recently, Liu and Hua [17] proposed two new numerical methods for the study of the 3D semi-periodic line-contact, by considering the contact periodic in the length direction. This author [18] also applied spectral methods to simulate the contact process of viscoelastic materials in finite length line-contacts. Reviews on the application of the FFT in tribological modelling can be found in $[10,19]$. In the light of these recent developments in the spectral analysis of contact problems, two modifications of the state-of-the-art Discrete Convolution Fast Fourier Transform (DCFFT) algorithm are advanced in this paper. Whereas the DCFFT was previously developed for purely nonperiodic contact problems, this paper explores the application of the latter technique to hybrid contact scenarios, with non-periodic behaviour in one direction, but that can be treated as periodic in the other direction. The advanced algorithms were validated against Hertz analytical solutions for the 2D smooth contact of infinite cylinders.

\section{CONTACT MODEL OVERVIEW}

The plain-strain assumption is an efficient way to reduce the line-contact problem model to a $2 \mathrm{D}$ analytical model whose solution can be achieved more easily than its 3D counterpart, e.g., in the frame of the Hertz contact theory. Such simplification may be feasible over the majority of the length of the cylinder, but it cannot however account for the stress concentrators occurring close to the ends. To study the edge effect and the influence of the roller crowning on the aforementioned stress riser, a 3D contact problem must be solved. This is also the case with deterministic contact simulations that employ real surface topography measured by optical measurement systems. The solution of the $3 \mathrm{D}$ contact problem with consideration of roughness in a deterministic manner was achieved by Polonsky and Keer [20]. The algorithm is based on a modified conjugate gradient scheme, which derives simultaneously the pressure distribution and the contact area, by iterating discrete pressure elements as the unknowns of a linear system of equations, whose size is also a priori unknown. The condition for the latter solution process is that the displacement induced by arbitrary pressure distribution can be efficiently computed.

The need for algorithmic efficiency is more critical in case of rough contacts with high density of grids on the contact region. Whereas smooth contact geometries can be simulated with as low as $10^{2}$ point on the contact area, rough surfaces require at least $10^{6}$ discrete heights to capture the specific topography features. An additional challenge appears in the numerical study of the 3D line-contact depicted in Figure 1, as the contact area is much smaller in the contact width direction than in the contact length. It should be noted that the grid step should be comparable in the two directions, and the consideration of a $N$ points grid results in a linear system with $N^{2}$ unknowns. Considering that at least $10^{3}$ grids may be required in the width direction to properly describe microtopography, the size of the linear system to be solved rises rapidly beyond the computational power of modern computers.

An alternative approach is followed in this paper, depending on the periodic nature of any microtopography resulted from common manufacturing processes. A slice of the contacting cylinder is chosen as a representative target domain, as shown in Figure 1, and the rest of the contact length is assumed to match the target domain in a periodical manner. This means that all problem distributions are assumed periodical in the contact length direction, with a period equal to the extent of the target domain. The latter should be chosen so that the representative features of the surface microtopography are well represented within. Although the calculations are performed on one period only, the results are thus valid for the periodically extended windows as well.

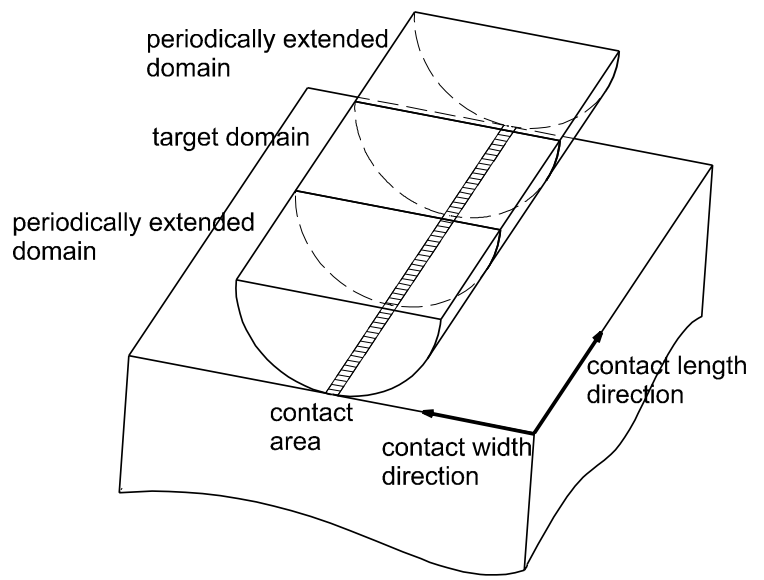

Fig. 1. Schematic of the semi-periodic 3D line-contact

One important feature of the contact process, and in the same time the main difficulty in achieving the contact solution, is that the displacement at any point on the contact surface depends on the pressure distribution in the whole contact region. Therefore, the solution of an integral equation in pressure is required for the contact 
solution. The latter equation can be turned into a linear system with a set of discrete pressure elements as unknowns. It is convenient to arrange these unknowns in a matrix $p_{i, j}$, with $i=1 \ldots N_{\text {length }}$ and $j=1 \ldots N_{\text {width }}$ the numbers of grids considered in each direction. The grid steps on each of the two directions should be comparable for accurate description of surface topography, although this is not a method requirement.

With the aid of the so-called influence coefficients [20] or influence functions [21], the integral expressing the displacement $u$ induced by contact pressure can be digitized into a $2 \mathrm{D}$ (for a $3 \mathrm{D}$ contact model) multi-summation:

$$
u_{i, j}=\sum_{k=1}^{N_{\text {lenggh }}} \sum_{\ell=1}^{N_{\text {widhl }}} K(i-k, j-\ell) p_{k, \ell},
$$

in which the liaison between the observation node $(i, j)$, $i=1 \ldots N_{\text {length }}, j=1 \ldots N_{\text {width }}$ and the excitation node $(k, \ell)$ is achieved through the influence coefficient $K(i-k, j-\ell)$. The latter expresses directly the individual contribution of the pressure node $p_{k, \ell}$ to the displacement in the observation node $(i, j)$. By varying the position of both the excitation and the observation nodes, the displacement throughout the whole computational domain is calculated based on contributions of all pressure nodes. The computation of the influence coefficients, detailed in [20], shows that, except for a constant related to the contact compliance, $K(i-k, j-\ell)$ depends only on the distance between the excitation and the observation point. This results in a reduction of the different influence coefficients to be computed. As an example, for a one-dimensional grid with $N+1$ points and $\Delta$ mesh interval, there exist only $2 N+1$ different influence coefficients, ranging from $K(-N \Delta)$ to $K(N \Delta)$. The former stands for the contribution of the excitation node $i=1$ to the observation point $j=N+1$, whereas for the latter the two nodes are simply reversed.

The problem of high stress gradients in a confined domain is solved in finite element analysis by imposing variable step meshes, with finer mesh in the region of interest. However, this approach is not feasible in case of the current method, as the number of different distances between the excitation sources and measured effect locations, and implicitly the number of different influence coefficients, increases dramatically, thus making the multi-summation process computationally inefficient. Consequently, a uniform mesh should be imposed, with intervals that can differ on each direction. A non-conventional, yet very efficient way of performing the multisummation (1), namely the Discrete Convolution Fast Fourier Transform (DCFFT), was advanced [11,12] for non-periodic contact problems, and it will be modified in the following section to allow semiperiodic contact scenarios.

\section{APPLICATION OF DCFFT TO SEMI- PERIODIC CONTACT PROBLEMS}

The main idea of the DCFFT [11] is to treat the multisummation (1) as a discrete convolution that can be calculated as an element-wise product in the frequency domain, as postulated by the convolution theorem. The computational advantage is significant: for series with $N$ terms, the order of operations for (1) is $O\left(N^{2}\right)$, which is reduced in the frequency domain to $O(N)$ as a termby-term multiplication. Considering that the transfer to and from the frequency domain can be achieved with the fast Fourier transform in $O\left(N \log _{2} N\right)$, the latter results as the overall computational complexity of the DCFFT. The main steps for the DCFFT are restated here for completeness, whereas the full details needed for the algorithm implementation in a computer code can be found in [11].

1. Compute the influence coefficients and treat the resulting series with zero-padding and wrap-around order, to accomplish the required circular nature [19] of the convolution series.

2. Digitize the pressure input and preform zero-padding in a domain twice (in every direction) the original one.

3. Transfer both pressure and influence coefficients series (from the space domain) to the frequency domain via FFT.

4. Perform element-wise multiplication between series of complex numbers, thus obtaining a spectral representation of the required displacement.

5. Transfer the latter series back to the space domain by inverse FFT and discard the results in the zeropadded domain.

The resulting series is the displacement induced by the input pressure. As discussed in $[11,12]$, the DCFFT is designed to solve a non-periodic scenario, i.e., only the contribution of the pressure distribution in the considered domain is accounted for in displacement computation. In case of a semi-periodic line-contact, although the contact problem is solved on the target domain only, the calculated displacement for the latter domain should ideally take into account the whole pressure distribution, which is periodical in one direction and non-periodical in the other.

A first way to deal with this semi-periodicity is to modify the step 2 of the algorithm by mimicking a semi-periodic pressure: in the contact width direction, the pressure should be zero-padded as in the original algorithm (Figure 2(a)), whereas in the contact length direction the pressure should be duplicated as shown in Figure 2(b). This simple technique proved its efficiency in dealing with periodicity in the elasto- 
plastic contact of nominally flat surfaces [16]. The pressure from the target domain is thus zero-padded in the direction in which the problem is non-periodic, and copied (shifted) in the periodic direction. The rest of the algorithm is kept replicated exactly. This trivial modification has non-trivial effects on the algorithm outcome, as shown by the comparison performed in the results section. The method is particularly advantageous because the virtual domain for the displacement calculation remains the same, and consequently the computational effort is not increased.

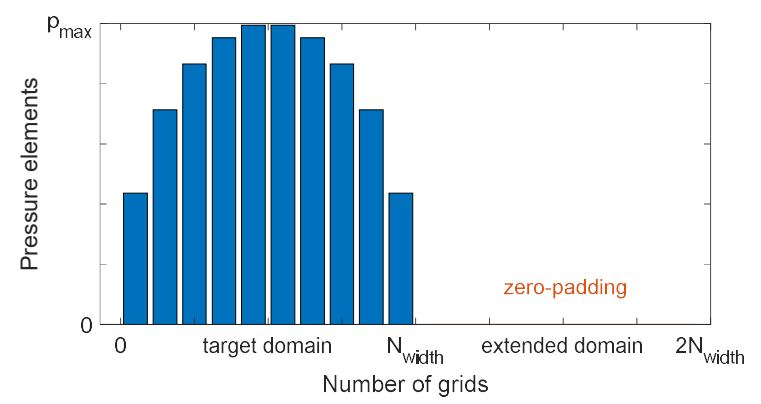

(a)

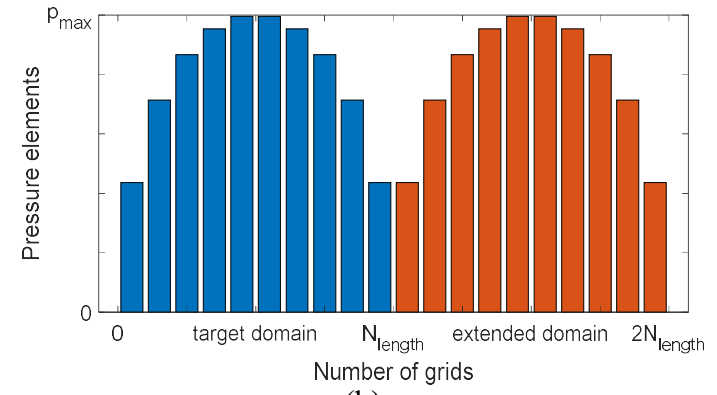

(b)

Fig. 2. Pressure treatment in modified DCFFT: a) contact width direction (non-periodic); b) contact length direction (periodic)
A second algorithm variant can be constructed from the definition of the influence coefficient itself. The contribution of the neighboring (i.e., lateral in the direction of the contact length direction) pressure period to the displacement in the target domain can be expressed as:

$$
\Delta u_{i, j}=\sum_{k=1}^{N_{\text {length }}} \sum_{\ell=1}^{N_{\text {vidh }}} K\left(i-k+N_{\text {length }}, j-\ell\right) p_{k+N_{\text {length }}, \ell} .
$$

Considering pressure periodicity,

$$
p_{k+N_{\text {lengh },}, \ell}=p_{k, \ell}, \quad k=1 \ldots N_{\text {length }}, \quad \ell=1 \ldots N_{\text {width }} .
$$

In light of the superposition principle, (2) can be superimposed on (1) and the pressure factorized, resulting in a summation of influence coefficients multiplied by the pressure in a single period:

$u_{i, j}=\sum_{k=1}^{N_{\text {leagh }}} \sum_{\ell=1}^{N_{\text {with }}}\left(K(i-k, j-\ell)+K\left(i-k+N_{\text {length }}, j-\ell\right)\right) p_{k, \ell}$.

Moreover, if $n$ pressure periods are considered on each side of the target domain, in the contact length direction, and the summation operators are interchanged, the displacement in the target domain results as:

$$
u_{i, j}=\sum_{k=1}^{N_{\text {lengh }}} \sum_{\ell=1}^{N_{\text {widh }}}\left(K(i-k, j-\ell)+\sum_{m=1}^{n} K\left(i-k+m N_{\text {length }}, j-\ell\right)+\sum_{m=1}^{n} K\left(i-k-m N_{\text {length }}, j-\ell\right)\right) p_{k, \ell} .
$$

The latter is similar in structure to (1) and consequently can be treated with the same type of algorithm, namely the DCFFT, but with a modified $K$ matrix. The influence coefficients in a domain $(2 n+1)$ times the target domain (in the periodic direction) should first be calculated. The summation in (5) should then be performed, giving an influence coefficients series that also takes into account the contribution of the $2 n$ adjacent pressure periods. The latter series is then inputted in the DCFFT algorithm. It was found by numerical experimentations presented in the next section that for $n>6$, this superposition technique gives very similar results to the pressure duplicated padding method.

It can be argued that the second approach decreases the computational efficiency because of the need to calculate the influence coefficients in a larger domain.
However, the computational effort for influence coefficients assessment is much smaller than the one for the multi-summation (or convolution) itself. On the other hand, in the solution [20] of the contact problem, the influence coefficients only need to be computed once before the start of the iterative process, whereas the convolution must be performed repeatedly, two times per iteration. Consequently, both methods make good candidates for the numerical simulation of contact problems that can be approximated as periodic in one direction but non-periodic in the other.

\section{RESULTS AND DISCUSSIONS}

The proposed approach if first applied to compute the displacement induced by a periodic distribution of semielliptic pressures such as the one resulting in a Hertz point contact. A single pressure, fully described by the 
contact radius $a_{H}$ and the central maximum pressure $p_{H}$, is centered in a square target computational domain of side lengths $3 a_{H}$. The maximum pressure $p_{H}$ is thus located in the center of the square, where $x_{\text {width }}=x_{\text {length }}=0$. The pressure is then assumed periodical in the $x_{\text {length }}$ direction, with a period of $3 a_{H}$, i.e., the length of the target domain. The induced displacement is calculated with the classic DCFFT, involving zero-padding in all directions, as well as with duplicated padding of pressure in the periodic direction and zero-padding in the non-periodic one, as depicted in Figure 3. The numerical predictions are compared in Figure 4, in which the analytical Hertz solution [21] for a point contact is also included for reference. Dimensionless displacement is defined as ratio to the maximum central displacement, i.e., the rigid-body approach in a point contact $\delta=u(0,0)$.

The plotted pressure distributions show that in the plane $x_{\text {length }}=0$ (Figure 4(a)), the duplicated padding shifts the displacement in the normal direction, whereas in the plane $x_{\text {width }}=0$, the shift is accompanied by a modification in curvature that is more visible at the periphery. In the latter location, the contribution of the neighboring periods is more consistent, as clearly predicted by the numerical simulation.

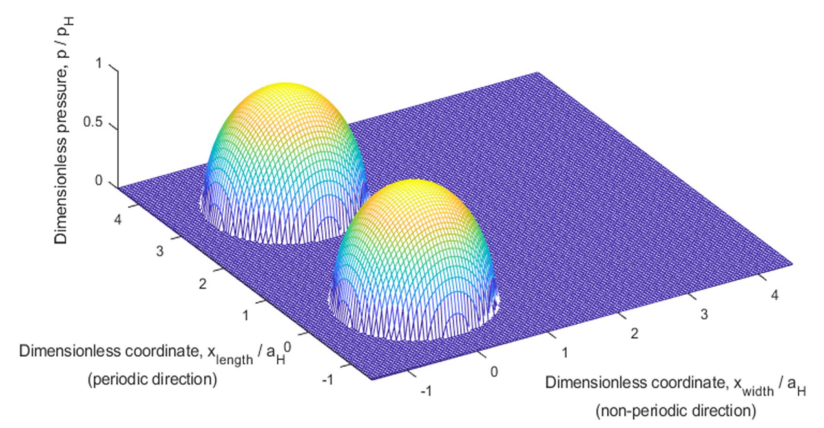

Fig. 3. Duplicated padding and zero-padding of a Hertz-type pressure

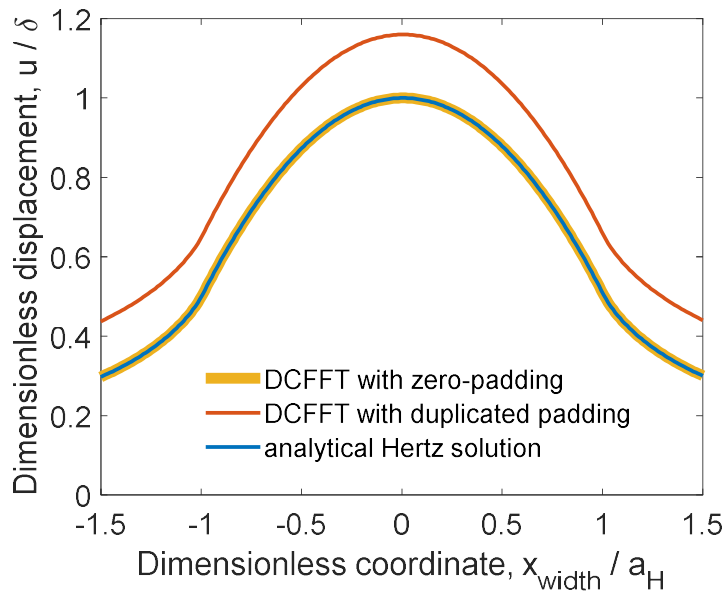

(a)

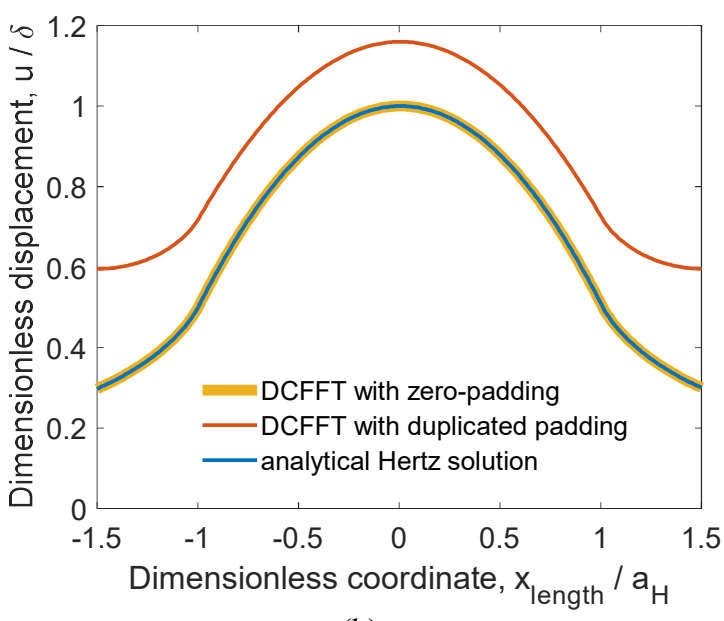

(b)

Fig. 4. Influence of duplicated padding in DCFFT displacement calculation: a) non-periodic direction (contact width); b) periodic direction (contact length)

The proposed algorithms are then used to replicate the Hertz solution for the contact of infinitely long cylinders. Despite the infinite nature of the problem, a finite longitudinal domain is chosen as target domain, whereas in the transversal direction the side length is $2.4 a_{H}$, with $a_{H}$ the contact semi-width. It was verified by numerical trials that the results are independent of the domain extent in the length direction. The latter extent is also the size of the window whose periodic lateral multiplication replicates the infinite contact length. The analytical dependencies of the model parameters can be found in [21]:

$$
a_{H}^{2}=\frac{4 W R}{\pi E} ; \quad p\left(x_{\text {width }}\right)=\frac{2 W}{\pi a_{H}^{2}} \sqrt{a_{H}^{2}-x_{\text {width }}^{2}} ; \quad p_{H}=\frac{2 W}{\pi a_{H}}
$$

where $R$ is the relative curvature related to the radii $R_{i}, i=1,2$ of the two cylinders, $1 / R=1 / R_{1}+1 / R_{2}, E$ is the composite modulus, $E=\left(1-v_{1}^{2}\right) / E_{1}+\left(1-v_{2}^{2}\right) / E_{2}$, with $E_{i}$ and $v_{i}, i=1,2$ the Young moduli and the Poisson's ratios of the contacting materials, $p_{H}$ is the maximum pressure, and $W$ is the compressive load per unit axial length. The target domain was meshed with $128^{2}$ grids, and a value $m=6$ was imposed in the summation (5). Figure 5 shows that the analytical relations (6) are replicated numerically with either algorithm variant. The good agreement suggests that the proposed methods can handle the considered class of problems with increased precision and computational efficiency. 


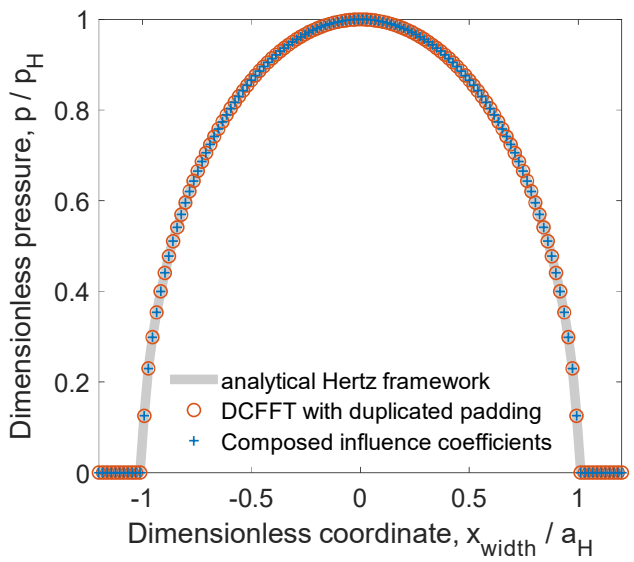

Fig. 5. Transversal pressure distribution in the contact of infinitely long cylinders

\section{CONCLUSIONS}

Two 3D contact models that allow for the analysis of rough contacts involving deterministic data resulting from profilometer measurements of machine elements are advanced in this paper. The methods strong points are the robustness of the influence coefficients technique and the efficient computation of displacement in the frequency domain. The linecontact problem, usually treated with $2 \mathrm{D}$ analytical or numerical models within the plain-strain assumption, is tackled here with a 3D model supported by the assumption of periodicity in the contact length direction. With this approximation, the meshing in the latter direction is restricted to a finite domain whose periodical extension replicates the real contact surface. A state-of-the-art algorithm for displacement computation in non-periodic contact problems, namely the Discrete Convolution Fast Fourier Transform, is adapted for a semi-periodic approach. A first algorithm variant employs a special padding technique for pressure that mimics periodicity, whereas a second one uses superposition principle to define composed influence coefficients that take into account the contribution of the adjacent pressure periods. In this manner, the calculated displacement comprises contributions from the pressure elements outside the considered target domain. Both techniques conserve the method computational efficiency, allowing for finer meshes in the contact solution. The performed numerical simulations confirm the accuracy of the program predictions against the Hertz analytical framework. The theoretical pressure distribution in the contact between infinite cylinders is precisely reproduced by the computer program with a finite $2 \mathrm{D}$ target domain. As the numerical approach promotes an essentially three-dimensional study, the influence of roughness or texture of real surfaces in a line-contact can be further studied with a computational effort comparable to that for a concentrated contact case.

\section{ACKNOWLEDGEMENT}

This work was partially supported from the project "Integrated Center for Research, Development and Innovation in Advanced Materials, Nanotechnologies, and Distributed Systems for Fabrication and Control", Contract No. 671/09.04.2015, Sectoral Operational Program for Increase of the Economic Competitiveness co-funded from the European Regional Development Fund.

\section{REFERENCES}

1. Gupta P K and Walowit J A, (1974), ASME J. Lubr. Technol. 96 250-257.

2. Webster M N and Sayles R S, (1986), ASME J. Tribol. 108 314-320.

3. Merriman T and Kannel J, (1989), ASME J. Tribol. $11187-94$

4. Elsharkawy A A and Hamrock B J, (1993), ASME J. Tribol. 115 237-245

5. Ju Y and Farris T N, (1996), ASME J. Tribol. 118 320-328.

6. Mao K, Sun Y and Bell T, (1996), Tribol. Trans. 39 416-424.

7. Mihailidis A, Bakolas N, and Drivakos N, (2001), Wear 249 546-556.

8. Bucher F, Knothe K, and Theiler A, (2002), Wear $253204-218$.

9. Kadiric A, Sayles R S, Zhou X B, and Ioannides E, (2003), ASME J. Tribol. 125 720-730.

10.Liu S, Chen W W, Hua D, and Wang Q, (2007), Tribol. Int. 40 1284-1293

11.Liu S B, Wang Q and Liu G, (2000), Wear 243 101111.

12.Liu S B and Wang Q, (2002), ASME J. Tribol. 124 36-45.

13.Polonsky I A and Keer L M, (2000), ASME J Tribol. $12236-41$.

14.Liu S and Wang Q, (2005), ASME J. Appl. Mech. 72 871-878.

15.Spinu S, (2014), J. Balk. Tribol. Assoc. 20 63-74.

16.Chen W W, Liu S, and Wang Q, (2008), ASME $J$. Appl. Mech. $\mathbf{7 5} 011022$.

17. Liu S, Hua D Y, (2009), ASME J. Tribol. 131021408.

18. Spinu S, (2018), Tribology in Industry 40 538-551.

19.Wang Q J, Sun L, Zhang X, Liu S, and Zhu D, (2020), Front. Mech. Eng. 661.

20. Polonsky I A and Keer L M, (1999), Wear $231206-$ 219.

21.Johnson K L, (1985), Contact Mechanics (Cambridge: University Press).

Received: June 25, 2021 / Accepted: December 15, 2021 / Paper available online: December 20, 2021 (C) International Journal of Modern Manufacturing Technologies. 